\title{
Éditorial: À propos des odes d'Horace et du fait d'être un examinateur
}

Il y a déjà quelques semaines que j'ai vécu une expérience qui arrive très rarement au cours d'une carrière universitaire. On m'a demandé d'agir à titre d'examinateur externe pour un mémoire de maîtrise au Département des lettres classiques de mon université. Comme la plupart d'entre vous, je crois que je suis une personne assez cultivée, mais voulez-vous bien me dire en quoi consiste mon savoir sur les classiques et pourquoi demanderait-on à quelqu'un qui fait de la recherche sur le vieillissement, l'accès aux soins formels et informels et le rôle des bénévoles de faire partie du jury d'examen d'une thèse sur ce sujet. La thèse s'intitulait "Old Age, Ageing and Death in Horace's Odes (Vieillesse, vieillissement et mort dans les odes d'Horace)», et avait été rédigée par Aara Macauley. Son directeur de thèse $\mathrm{m}^{\prime} \mathrm{a}$ dit qu'il tentait souvent de sélectionner des personnes à l'extérieur du domaine des sciences humaines pour l'examen des thèses de ses étudiants. Je peux confirmer que cette thèse était un texte de recherche vraiment exceptionnel et très agréable à lire, mais il m'a aussi porté à réfléchir aux défis en tant que rédacteur en chef de La Revue canadienne $d u$ vieillissement $(R C V)$.

Les deux plus grands défis que je ne m'attendais pas à affronter lorsque j'ai accepté le poste que j'occupe sont d'une certaine façon les mêmes que ceux que j'ai rencontrés lorsque j'ai lu la thèse d'Aara. Pendant la lecture, je me demandais souvent quelles questions je pourrais bien poser en rapport avec le texte. D'une façon presque similaire, chaque fois qu'un manuscrit arrive à mon bureau et que je le lis, je dois me demander s'il est assez bon pour que je l'envoie à un rédacteur de section pour révision et, dans l'affirmative, à quel rédacteur de section je dois l'envoyer. Même si je ne suis pas un spécialiste des odes d'Horace, j'ai trouvé assez de correspondances entre ses descriptions du vieillissement et les attitudes envers les personnes âgées de la Rome d'Octave pour pourvoir former des questions basées sur ma vision contemporaine de ces questions. De façon similaire, même si je ne suis pas un spécialiste de tous les aspects de la gérontologie, je suis heureux de dire qu'il m'arrive rarement de décider qu'il ne vaut pas la peine d'envoyer le manuscrit à un rédacteur de section et, la plupart du temps, les rédacteurs de section ne se demandent pas pourquoi je le leur ai envoyé.
Le second défi est que tous les examinateurs doivent décider de l'issue du travail écrit et de l'examen oral. Dans le cas d'Aara, ce fut très facile. La thèse était un texte de recherche exceptionnel. À titre de rédacteur en chef, j'ai à décider du sort de chaque manuscrit en me basant sur le dur travail des réviseurs et des éditeurs de section. Les éditeurs de section présentent des recommandations et, lorsqu'elles sont négatives, leur décision, je le sais pertinemment, n'a pas été facile à prendre. À mon tour, j'ai à communiquer les résultats à l'auteur. De plusieurs façons, les examinateurs, les rédacteurs de section et moi-même formons comme un jury de thèse. Même lorsque je refuse un manuscrit, j'espère que l'auteur tirera des leçons des rapports que nous lui envoyons, qu'il retravaillera son manuscrit, et qu'il trouvera une publication appropriée pour sa recherche, même si ce n'est pas le $R C V$.

$\mathrm{Si}$ j'avais à donner une note à mon propre travail comme votre rédacteur en chef depuis les deux dernières années, je pencherais probablement vers une note dans les « $\mathrm{B} »$. Je suis fier de certaines des expériences auxquelles nous nous sommes livrés lors de la publication d'articles et d'éditoriaux qui s'écartaient un peu de la norme, des changements que nous avons apportés à la structure du comité de rédaction, et du rôle que nous avons joué auprès $\mathrm{du}$ Congrès international francophone de gérontologie et gériatrie en publiant ses résumés dans un supplément de la revue. Passer à la présentation électronique n'a pas progressé aussi rapidement que je l'aurais souhaité et, peut-être pour moi et j'en suis persuadé pour plus d'un auteur en puissance a été le temps que cela prend parfois pour leur faire parvenir un premier jeu de rapports des examinateurs. Je n'ai pas de solution facile à ce problème, parce qu'il découle de plus en plus sur la difficulté que nous avons à retenir les services de chercheurs qualifiés pour agir comme examinateurs et à obtenir que leurs rapports soient livrés dans les délais prévus.

Pour revenir à la thèse d'Aara, même s'il aurait été facile pour moi de décliner l'invitation à examiner la thèse parce que je n'étais pas certain que j'étais la personne la plus qualifiée comme examinateur externe et en raison des nombreuses autres tâches qui me sont dévolues à mon université, j'ai dit oui. J'ai beaucoup appris. J'ai trouvé dans la thèse 
quelques problèmes de rédaction et j'avais aussi quelques questions à poser à Aara.

J'espère que la prochaine fois qu'on vous demandera de faire partie de l'équipe d'examen d'un article pour la $R C V$, même si vous vous demandez si vous êtes la meilleure personne ou si vous avez d'autres tâches qui vous pressent, vous direz «oui» et que, comme moi, vous réaliserez que, par notre participation à tous les aspects du processus de recherche, nous apprenons constamment, contribuant ainsi à la $R C V$, et au perfectionnement de la gérontologie en tant que discipline.

Comme toujours, veuillez communiquer avec moi à rosenber@post.queensu.ca si vous avez des observations au sujet de cet éditorial ou sur tout autre aspect de la $R C V$.

Mark Rosenberg

Rédacteur en chef 


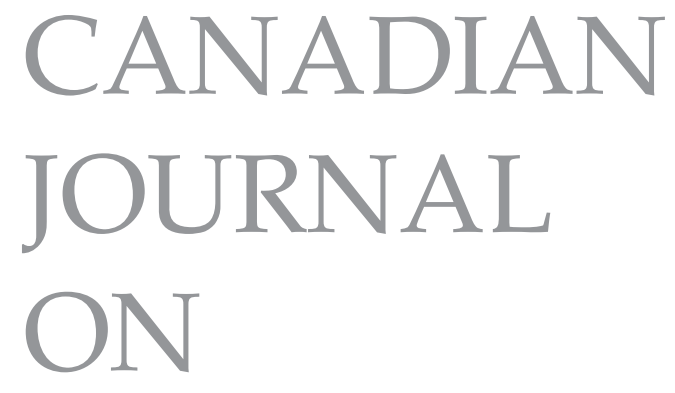

AGING

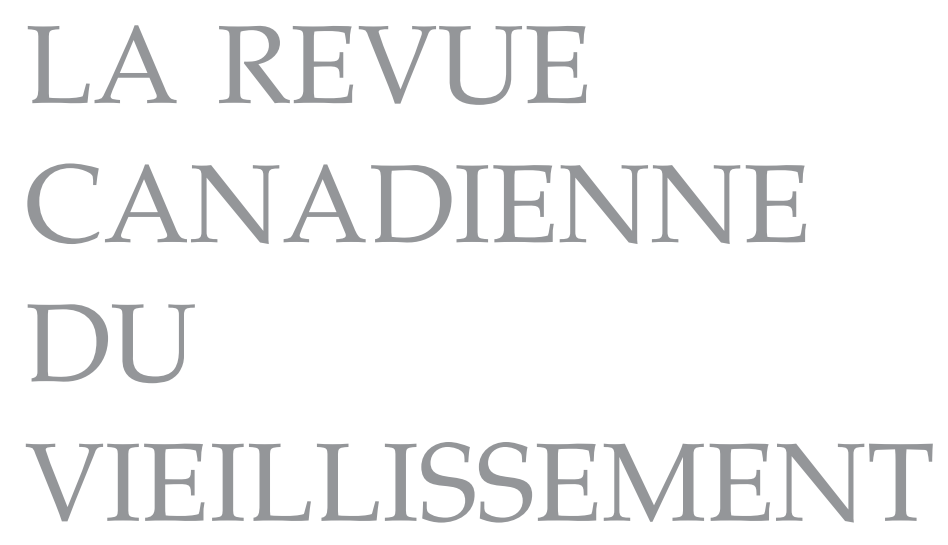

